\title{
Basophil Reactivity as Biomarker in Immediate Drug Hypersensitivity Reactions-Potential and Limitations
}

\author{
Markus Steiner ${ }^{1,2}$, Andrea Harrer ${ }^{1,3}$ and Martin Himly ${ }^{1 *}$ \\ ${ }^{1}$ Division Allergy and Immunology, Department Molecular Biology, University of Salzburg, Salzburg, Austria, ${ }^{2}$ Laboratory for \\ Immunological and Molecular Cancer Research, Paracelsus Medical University, Salzburg, Austria, ${ }^{3}$ Department Neurology, \\ Paracelsus Medical University, Salzburg, Austria
}

\section{OPEN ACCESS}

Edited by:

José A. G. Agúndez, University of Extremadura, Spain

Reviewed by: Joan Bartra,

Barcelona University, Spain Adriana Ariza IBIMA-Regional University Hospital of Malaga-UMA, Spain

*Correspondence: Martin Himly martin.himly@sbg.ac.at

Specialty section: This article was submitted to Pharmacogenetics and

Pharmacogenomics,

a section of the journal

Frontiers in Pharmacology

Received: 28 April 2016 Accepted: 03 June 2016

Published: 17 June 2016

Citation:

Steiner M, Harrer A and Himly M (2016) Basophil Reactivity as Biomarker in Immediate Drug Hypersensitivity Reactions-Potential and Limitations.

Front. Pharmacol. 7:171 doi: 10.3389/fphar.2016.00171
Immediate drug hypersensitivity reactions (DHRs) resemble typical immunoglobulin E (lgE)-mediated symptoms. Clinical manifestations range from local skin reactions, gastrointestinal and/or respiratory symptoms to severe systemic involvement with potential fatal outcome. Depending on the substance group of the eliciting drug the correct diagnosis is a major challenge. Skin testing and in vitro diagnostics are often unreliable and not reproducible. The involvement of drug-specific lgE is questionable in many cases. The culprit substance (parent drug or metabolite) and potential cross-reacting compounds are difficult to identify, patient history and drug provocation testing often remain the only means for diagnosis. Hence, several groups proposed basophil activation test (BAT) for the diagnosis of immediate DHRs as basophils are well-known effector cells in allergic reactions. However, the usefulness of BAT in immediate DHRs is highly variable and dependent on the drug itself plus its capacity to spontaneously conjugate to serum proteins. Stimulation with pure solutions of the parent drug or metabolites thereof vs. drug-protein conjugates may influence sensitivity and specificity of the test. We thus, reviewed the available literature about the use of BAT for diagnosing immediate DHRs against drug classes such as antibiotics, radio contrast media, neuromuscular blocking agents, non-steroidal anti-inflammatory drugs, and biologicals. Influencing factors like the selection of stimulants or of the identification and activation markers, the stimulation protocol, gating strategies, and cutoff definition are addressed in this overview on BAT performance. The overall aim is to evaluate the suitability of BAT as biomarker for the diagnosis of immediate drug-induced hypersensitivity reactions.

Keywords: antibiotics, basophil activation test, biologicals, chemotherapeutics, fluoroquinolones, NMBAs, NSAIDs, RCM

\section{KEY PLAYERS IN THE IMMEDIATE-TYPE ALLERGIC EFFECTOR PHASE}

Key characteristic of allergic effector cells in immediate-type allergy is allergen-specific IgE bound to the high affinity IgE receptor, i.e., FceRI, on the cell surface. Capturing of allergens by surface IgE results in FceRI crosslinking and elicits the acute phase of the allergic response involving the sudden release of vasoactive mediators into the tissue and/or circulation. This sudden activation process and mediator release is termed anaphylactic degranulation (depicted in Figure 1A) and 
may induce life-threatening anaphylaxis (Hoffmann, 2015). Mast cells and basophils both share these key characteristics. Mast cells reside in the tissue and are considered primary allergic effector cells. Basophils are peripheral blood granulocytes, easily accessible via venipuncture and a well-established surrogate for allergy diagnosis (MacGlashan, 2013).

\section{THE BASOPHIL ACTIVATION TEST}

Basophil activation can be measured by flow cytometry and multicolor staining with fluorescent-labeled detection antibodies targeting specific identification and activation markers on the surface of basophils. The most common identification strategies use surface IgE, eotaxin CC chemokine receptor 3 (CCR3), the combination of interleukin 3 receptor alpha chain $\mathrm{CD} 123^{\text {high }}$ with human leukocyte antigen HLA-DR ${ }^{\text {neg }}$, the combination of prostaglandin $\mathrm{D}_{2}$ receptor CRTH-2 ${ }^{\text {high }}$ with CD $3^{\text {neg }}$, or the basophil-specific ectonuclease CD203c. Table 1 provides an overview of basophil identification strategies in drug hypersensitivity research which we numbered "strategy 1 " through "strategy 6" according to the frequency they have been used. For basophil activation the degranulation marker lysosomal-associated membrane glycoprotein-3 (LAMP-3), also termed CD63, or upregulation of CD203c are determined. Thus, changes in activation state can be quantified on a single cell basis. Degranulation means fusion of specific intracellular vesicles filled with preformed mediators, the so-called granules, with the plasma membrane and a transition of CD63 from inside out. The result is a sudden and pronounced rise, i.e., log-shift, of the fluorescence intensity signal, in the detection of surface CD63. Concomitantly, upregulation of CD203c has been observed which can be detected as significant increase in the mean fluorescence intensity signal of the CD203c detection antibody. Flow cytometry dot plots for both activation scenarios are depicted in Figure 1B. For further details about assay parameters of BAT the interested reader is referred to the comprehensive review of McGowan and Saini (2013).

In addition to anaphylactic degranulation another type of basophil activation termed piecemeal degranulation has been described (Dvorak, 2005). This alternative activation mechanism may also lead to altered surface expression of activation markers which can be assessed by BAT (Hausmann et al., 2009; MacGlashan, 2010). Consequently, BAT has been recognized as a promising tool for in vitro diagnosis of allergy or other hypersensitivity reactions including immediate adverse reactions to various drugs (Hoffmann et al., 2015).

\section{TECHNICAL ISSUES OF BASOPHIL ACTIVATION TESTING}

Usually BAT is performed from either heparinized, citrateor EDTA-anticoagulated whole blood collected from allergic/hypersensitive donors (Table 1). When EDTA is used as anticoagulant $\mathrm{Ca}^{++}$has to be supplemented to enable proper degranulation. For in vitro stimulation of basophils the samples are incubated with the allergen/drug or buffer only (negative control) for several minutes to hours at $37^{\circ} \mathrm{C}$. As positive control, anti-IgE antibodies, anti-FceRI antibodies, and formyl-methionine-leucine-phenylalanine (fMLF) are used. Latter represents an alternative degranulation/activation stimulus and is important to demonstrate basophil functionality in case of donors whose basophils fail to react in vitro to IgE-mediated pathway stimulation, so-called non-responders (Eberlein et al., 2010; MacGlashan, 2013).

Next, basophil identification and activation markers are stained with fluorescently labeled antibodies, subsequently erythrocytes are lysed. Depending on the protocol, staining can be performed during basophil stimulation in a single step. Upon flow cytometric acquisition of at least 200, in the optimal case 500-1000 basophils, activation marker expression is compared between buffer-treated samples and allergen-/drug-stimulated basophils. Different evaluation strategies are used. Some studies set the cut-off for spontaneously activated basophils arbitrarily at $5 \%$, whereas others use stimulation indices of \%CD63-/CD203cpositive cells, i.e., SI(\%), or mean fluorescence intensities (MFI) of activation markers, i.e., SI, compared to negative control (Table 1). For interpretation of BAT area under the dose curve (AUC) measurements have recently been postulated. These enable a combined evaluation of basophil reactivity, i.e., the dose (range) at which maximal response occurs, and basophil sensitivity, i.e., the dose at which half of the maximal response occurs. As the AUC representation incorporates partial energy, which may arise at high allergen concentrations, and can be calculated even in cases where responses do not fit the typical shape of dose-response curves, it is particularly useful for monitoring the efficacy in allergen-specific immunotherapy (Ebo et al., 2004; Hausmann et al., 2009; Hoffmann et al., 2015).

\section{BASOPHIL ACTIVATION TEST WITH DRUGS-BACKGROUND CONSIDERATIONS}

Small molecular weight drugs constitute haptens which are not capable of FcERI crosslinking themselves (hapten concept; Pichler et al., 2011). They require conjugation to carrier molecules (Figure 1A), usually abundant blood proteins, for eliciting an immune reaction in susceptible individuals. Moreover, reactive intermediates may be formed by drug metabolism (pro-hapten concept; Park et al., 1998; Naisbitt et al., 2000). Therefore, the use of drug metabolites and hapten-carrier conjugates has been promoted for the investigation of drug hypersensitivity reactions (Himly et al., 2003; Harrer et al., 2010; Steiner et al., 2011, 2014). Of note, in a case of propyphenazone (PP) hypersensitivity basophils reacted in BAT solely upon stimulation with the drugcarrier conjugate but not with pure PP (Steiner et al., 2014). Nevertheless, BAT is most frequently performed with solutions of plain drugs, a consequence of lacking knowledge in regard to relevant determinants, metabolic intermediates, their reactive functions, required linker length to the carrier molecule, and hapten orientation.

Alternative to the hapten and pro-hapten concepts in DHRs, the $\mathrm{p}$-i concept has become well-accepted, however, it primarily 


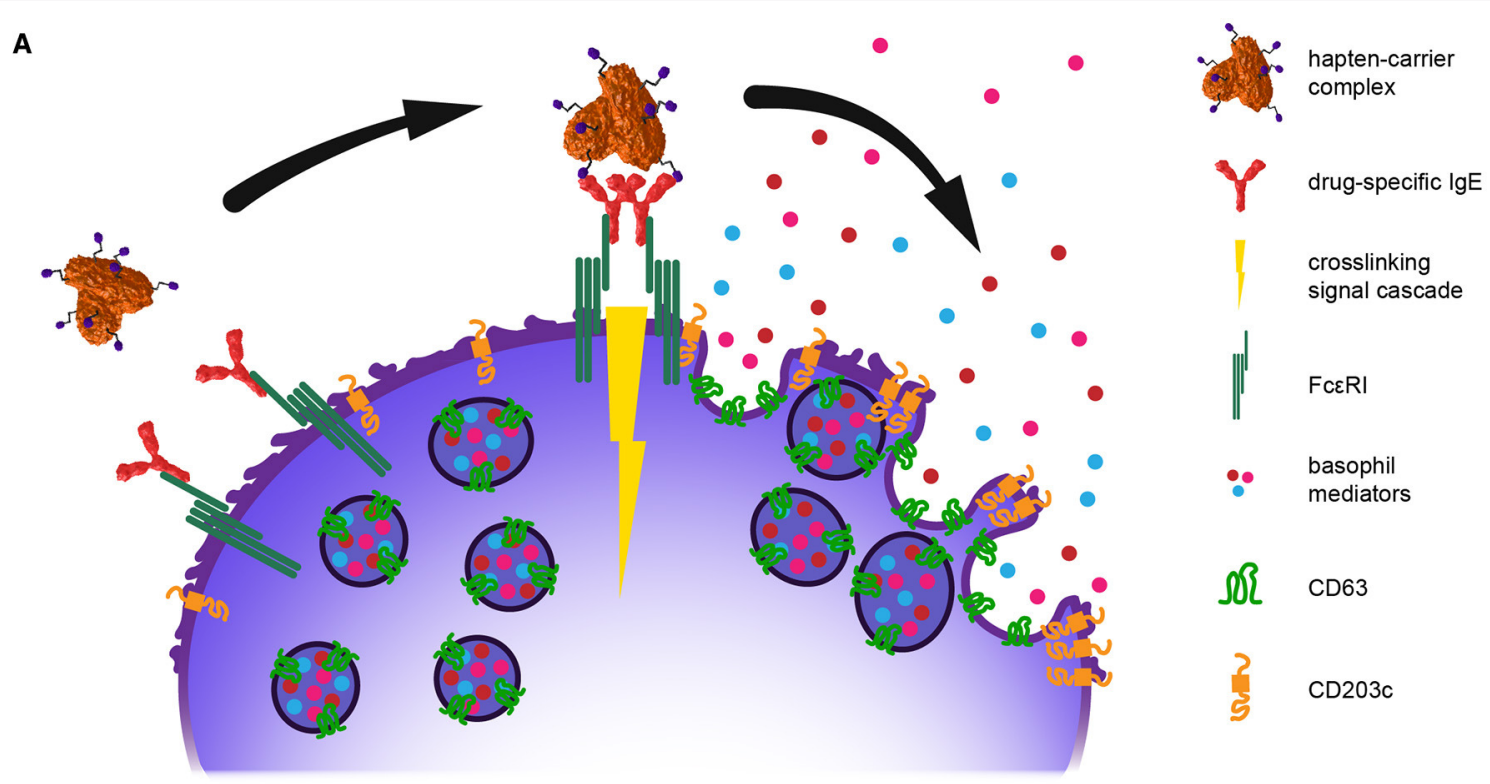

B CD63 surface expression

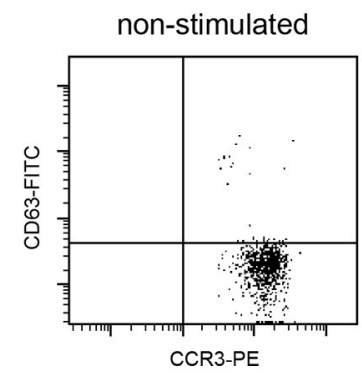

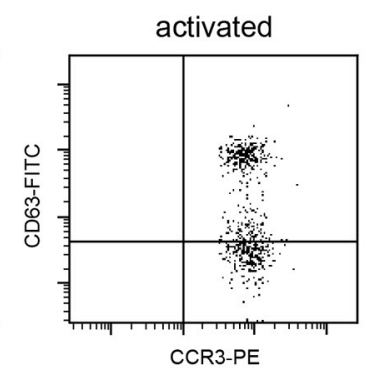
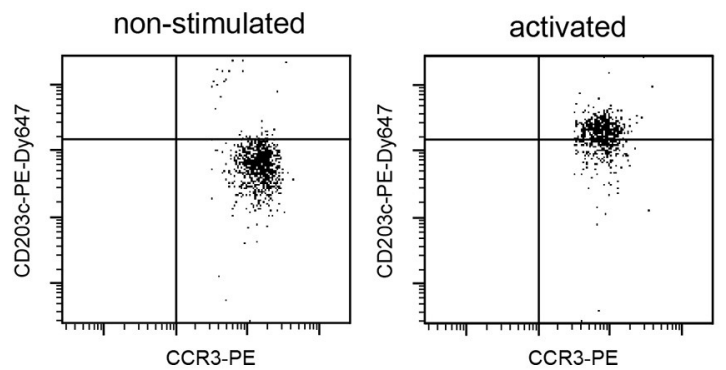

FIGURE 1 | (A) Schematic illustration of IgE-mediated cross-linking. Upon binding of the hapten-carrier complex to the drug-specific lgE captured on high affinity lgE receptor Fc\&RI, basophils react by degranulation and mediator release. CD63 is transferred from the vesicle to the plasma membrane and CD203c is upregulated, thus both serve as activation markers. (B) Flow cytometry dot plots of typical activation patterns for CD63 and CD203c; CCR3, basophil identification marker; PE, phycoerthrin label; FITC, fluorescein label; PE-Dy647, tandem label.

accounts for $\mathrm{T}$ cell-mediated delayed-type immune reactions against drugs such as lidocaine, sulfamethoxazole, lamotrigine, carbamazepine, p-phenylendiamine, etc. or against metals like in nickel contact dermatitis (Pichler et al., 2006). DHRs of this kind are elicited on a different time-scale, not discussed here, and an involvement of basophils is unlikely. A comprehensive overview can be gained from reviews by Pichler et al. (Pichler, 2003; Pichler et al., 2015).

\section{BAT FOR THE EVALUATION OF IMMEDIATE DRUG HYPERSENSITIVITY TO DIFFERENT DRUG CLASSES}

In the following paragraphs the suitability of basophil activation as a biomarker for evaluating immediate hypersensitivity reactions to different drug classes is discussed. The assay parameters used and activation patterns observed in the cited studies are summarized in Table $\mathbf{1}$.

\section{Basophil Activation in Antibiotics or Quinolone Hypersensitivity}

Immediate DHRs against beta-lactam antibiotics such as penicillin, amoxicillin, and cephalosporin have been broadly investigated and are most likely IgE-mediated. Diagnosis of betalactam allergy first place is based on skin prick and intradermal tests. Sensitivity of skin tests, however, does not exceed 50$70 \%$. The in vitro diagnostic method of quantifying beta-lactamspecific IgE antibodies (Mangodt et al., 2015) is an important complementary information. Clinically validated tests for drugspecific IgE, however, are difficult to develop, require complex coupling reactions for attaching the drug hapten onto a solid phase for antibody recognition, and are available only for a limited number of antibiotics. Instead, simple drug dilutions 
are used in BAT. It thus, appears a promising tool for in vitro diagnosis of beta-lactam allergy. Several groups investigated the applicability of BAT in the diagnostic management of betalactam allergy (Sanz et al., 2002; Gamboa et al., 2004a; Torres et al., 2004, 2010a, 2011; Abuaf et al., 2008; De Week et al., 2009; Eberlein et al., 2010; Garcia-Ortega and Marin, 2010). BAT performance, however, varied between groups with a median sensitivity of 50\% (range 22-55\%) and specificity ranging from 79 to $100 \%$. Quintessence of these studies is that BAT was superior to immunoassaying for drug-specific IgE, but not to skin testing. Importantly, skin testing and BAT do not necessarily corroborate each other. Positive skin testing has been confirmed by BAT only in about $50-60 \%$ of patients (De Week et al., 2009; Torres et al., 2010a), whereas up to one third of skin test-negative patients have been identified by BAT (De Week et al., 2009). The tenor across studies thus was that the role of BAT currently is complementary, respectively supplementary, in the diagnosis of beta-lactam allergy.

Factors possibly contributing to the observed variance in sensitivity of BAT may involve patients selection criteria such as severity of reactions and time elapsed since the reaction (optimum: 1-6 months), regional preferences in drug prescriptions, and whether the drugs tested allow identification of cross-reactors. Methodological variations such as differential activation times (range 20-40 min) and different activation markers (CD63 and/or CD203c) additionally complicate comparability of results.

One key question is why should basophils degranulate in an IgE-dependent fashion in vitro upon stimulation with a dilution of monomeric small antibiotic haptens? One theory is that the beta-lactam ring confers instability to the compound facilitating the conjugation of the drug to abundant blood proteins such as albumin, transferrin and/or immunoglobulins (Torres et al., 2016). Once attached to a carrier protein the side chain of the drug, the thiazolidine ring, or the conjugation site itself are immunogenic in susceptible individuals and capable of both, eliciting an IgE response and crosslinking of surface-bound IgE.

Beyond diagnostic purposes one important application of BAT is determining an IgE-mediated pathomechanism when drugspecific IgE cannot be evidenced. Wortmannin, for instance, is a strong inhibitor of phosphatidylinositol 3-kinase (PI3K) and inhibits basophil activation in response to FceRI crosslinking but not to stimulation with fMLF. In patients with selective allergy to the beta-lactamase inhibitor clavulanic acid, presence of drug-specific IgE was suspected but not detected. Using BAT an IgE-mediated pathomechanism was confirmed as BAT became negative upon stimulation with clavulanic acid in presence of wortmannin (Torres et al., 2010b).

BAT may be useful also in the diagnosis of immediate hypersensitivity reactions to quinolones. Diagnosis of quinolone allergy mainly relies on patient history and clinical manifestation. Skin tests are hampered by false positive reactions due to skin irritation rendering the positive predictive value of skin testing close to chance results. Drug-specific IgE have been reported, however, validated assays do not exist (Manfredi et al., 2004; Aranda et al., 2011; Mayorga et al., 2013). Aranda et al. showed both drug-specific IgE and dose-responsiveness in BAT for ciprofloxacin, moxifloxacin and levofloxacin, and inhibition of BAT positivity with wortmannin (Aranda et al., 2011). Moreover, they reported a higher sensitivity of BAT compared to IgE testing. Two out of seven studies evaluating BAT in quinolone hypersensitivity were negative with $0 \%$ sensitivity (Seitz et al., 2009; Lobera et al., 2010). The patient collectives of these two studies were quite small though as only 6 , respectively, 4 patients were included. Contrary, Rouzaire et al. (2012) tested 34 patients and reported a very good negative predictive value of BAT as quinolones were successfully reintroduced in 15 of the 17 patients (50\%) who tested negative in BAT. They emphasized the importance of negative BAT results in quinolone hypersensitivity as criteria for provocation test and thus the opportunity to possibly and safely reintroduce the drug (Rouzaire et al., 2012). Photodegradation could be one cause for false negative results. This was shown for moxifloxacin as positive results doubled when BAT was performed in the dark (Mayorga et al., 2013). Taken together, BAT appears helpful in the management of fluoroquinolone hypersensitivity.

\section{Basophil Activation in Neuromuscular Blocking Agent Hypersensitivity}

Anaphylactic episodes during general anesthesia have severe implications for the patient. Neuromuscular blocking agents (NMBAs) including rocuronium, vecuronium, atracurium, cisatracurium, and suxamethonium account for $>60 \%$ of such cases with a high degree of cross-reactivity within this drug group and even further to opioid antitussives such as codeine or morphine, with the existence of drug-specific IgE demonstrated (Baldo and Fisher, 1983; Vervloet et al., 1983; Sainte-Laudy et al., 2006; Ebo et al., 2007; Leysen et al., 2013). However, it has been recognized that $\mathrm{IgE}$ accounts for immediate DHRs against NMBAs only in $\sim 50 \%$ of cases. Therefore, a number of studies evaluated BAT protocols aiming at a more reliable before-hand screening tool (Monneret et al., 2000, 2002; Sudheer et al., 2005; Ebo et al., 2006; Kvedariene et al., 2006; Sudheer and Appadurai, 2007). Sudheer et al. (2005) compared CD63, CD203c, histamine release and skin testing for their predictive values. While specificities reached $100 \%$, the sensitivities of these four techniques determined for the whole NMBA group in their cohort of 21 patients resulted in 79, 36, 36, and 64\%, respectively. Other studies reported specificities for BAT based on CD63 expression of $>93 \%$ with sensitivities of $>54 \%$. As CD63 enables a better judgment of the type of basophil activation than CD203c, i.e., creating a log-shift in the flow cytometric dot plots, Ebo et al. (2006) were able to reach a sensitivity for DHR against rocuronium $>91.7 \%$ using $0.5 \mathrm{mg} / \mathrm{ml}$ of drug and setting the diagnostic threshold value at $4 \%$ to be most discriminative, as they determined from two-graph receiver operating characteristics. In a large study involving thorough diagnostic workup of 104 patients the same group (Leysen et al., 2011) obtained a positive predictive value of $98 \%$ for rocuronium using the combination of skin testing, BAT, and drug-specific IgE testing by ImmunoCAP. In their study, skin tests turned out most reliable, however, when these are negative, BAT is indicated. For assessment of cross-reactivity between rocuronium and 


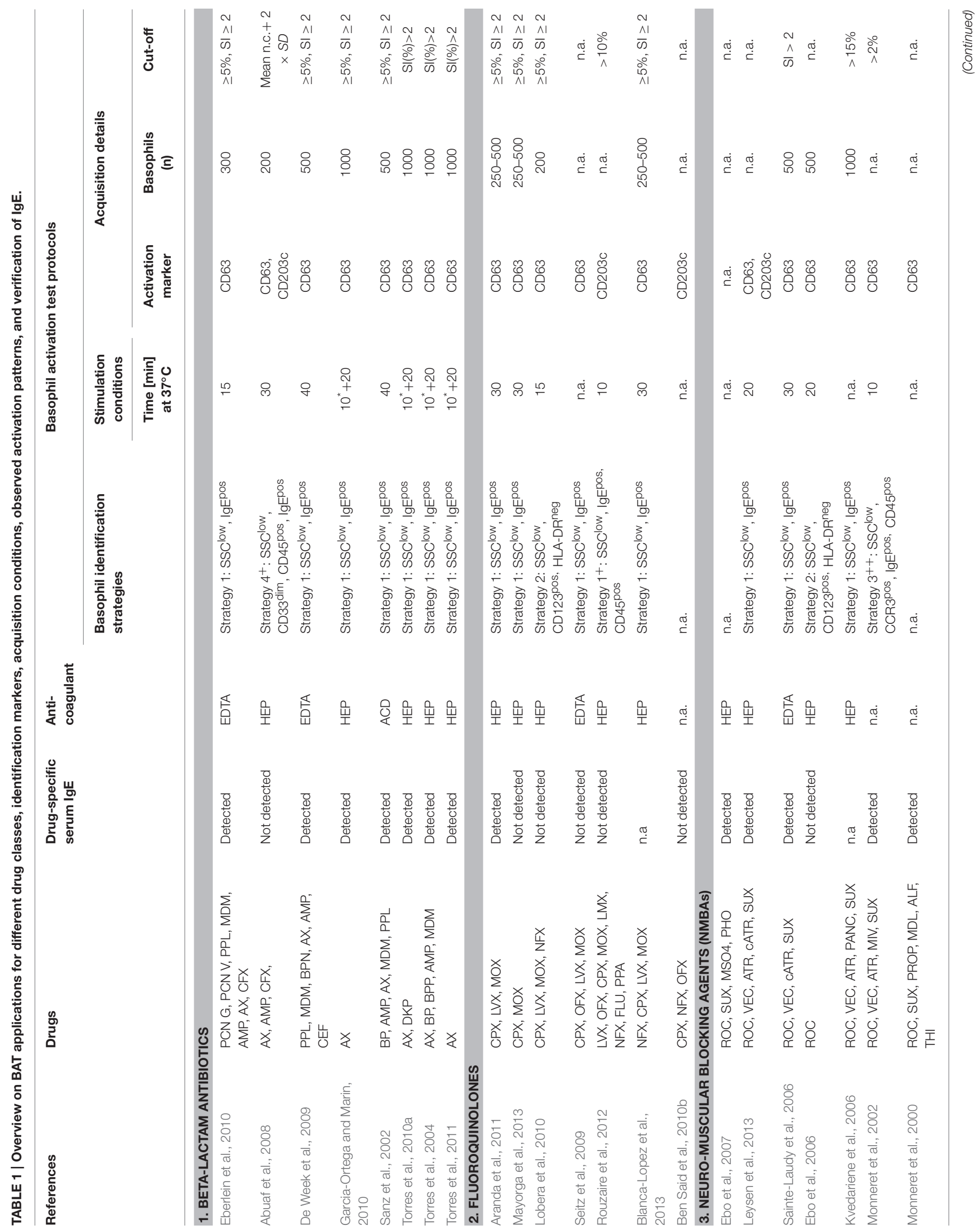




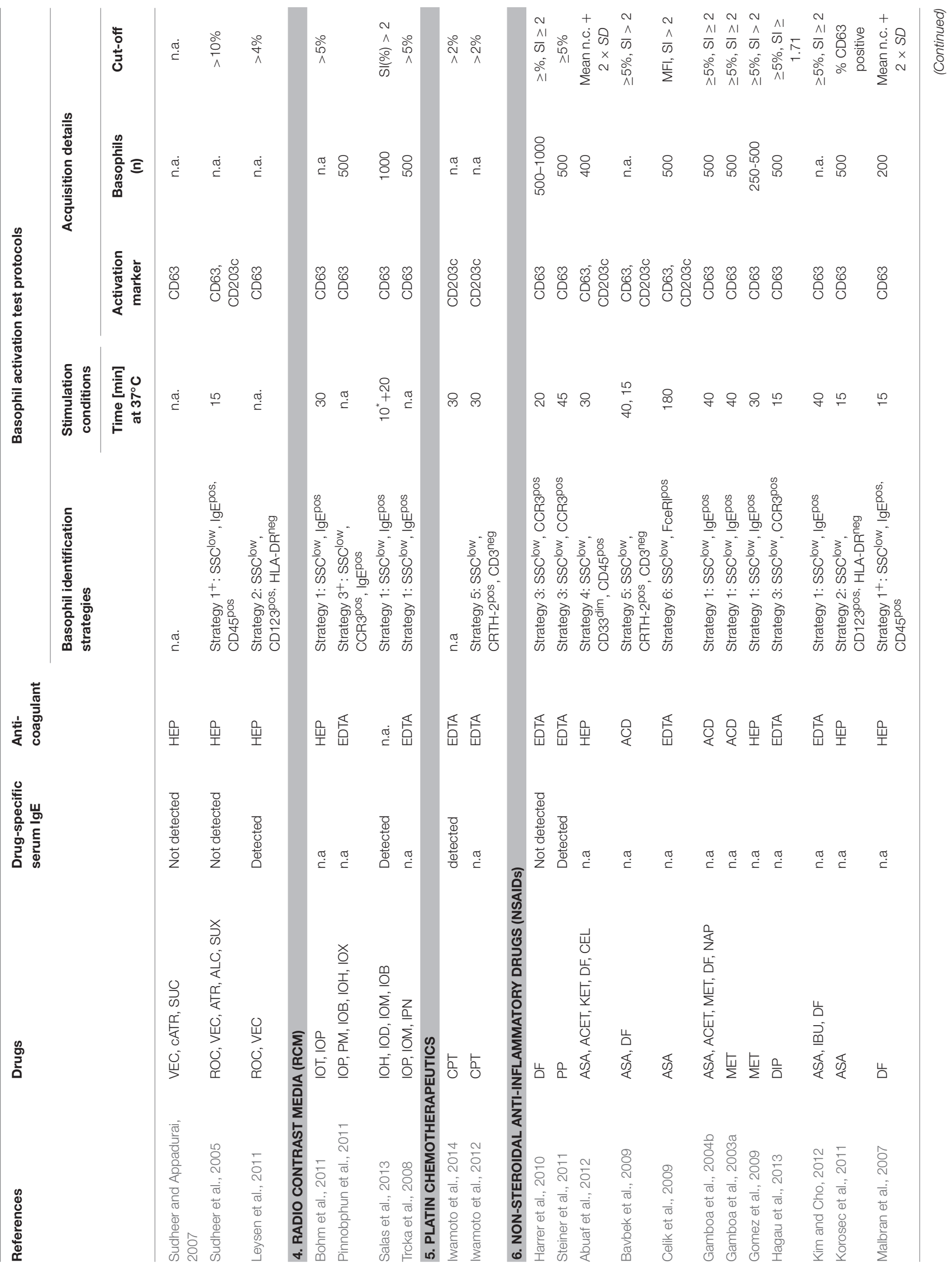




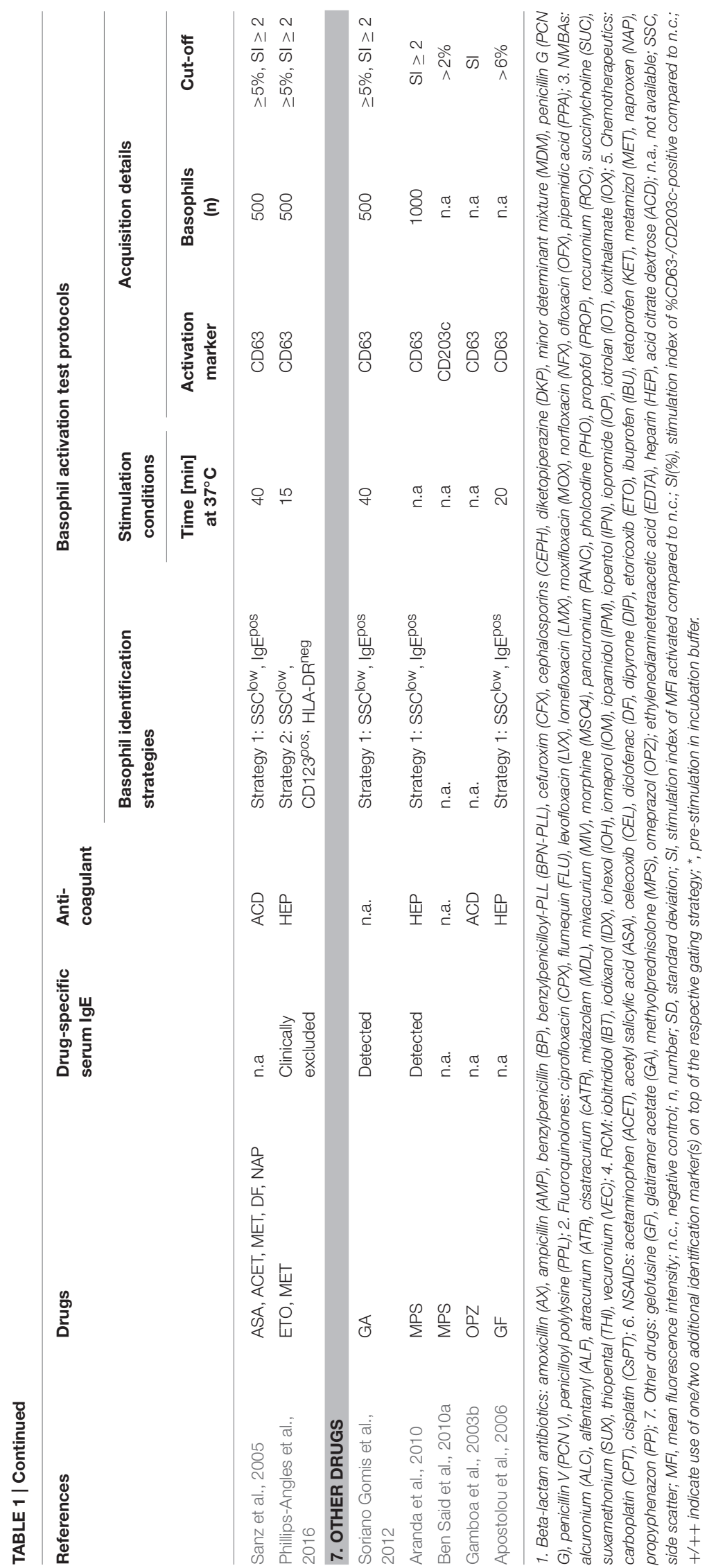


vecuronium, the two most often used NMBAs, BAT was found to complement skin tests well.

\section{Basophil Activation in Radiocontrast Media Hypersensitivity}

Immediate DHRs against non-ionic radiocontrast media (RCM) occur in $0.7-3.1 \%$ of patients, with ionic RCM being even more prevalent, i.e., up to $12.7 \%$ including mild reactions or reactions due to rapid intravenous infusion of these highly osmolar substances (Stellato et al., 1996). Nevertheless, $0.04 \%$ of patients experience severe reactions upon administration of non-ionic RCM (Wolf et al., 1989; Katayama et al., 1990; Lieberman and Seigle, 1999). Traditionally, RCM reactions have been considered as non-IgE-mediated, and the majority of DHRs indeed seem to result from RCM non-specifically binding to surface receptors or indirectly interfering with the complement or kinin cascades, but skin testing and BAT have been successfully applied more recently (Brockow et al., 2009; Javaloyes et al., 2012; Philipse et al., 2013). Still few anecdotal reports on RCM-specific IgE exist, therefore, some studies have sought to differentiate IgE- from non-IgE-mediated mechanisms, however, have not determined the presence of drug-specific IgE themselves. Moreover, a number of studies have evaluated BAT as a diagnostic tool for RCM hypersensitivity, however, giving variable experimental details, which makes it difficult to judge the mode of basophil activation (Trcka et al., 2008; Bohm et al., 2011; Pinnobphun et al., 2011; Salas et al., 2013). Presence of drug-specific IgE was not shown in any of the reports. Some studies demonstrated logshift activation of CD63 (Pinnobphun et al., 2011), while others did not (Bohm et al., 2011). Meta-analyzing the presented flow cytometric data some degree of inconsistency in the basophil activation profiles seems to be a common characteristic of immediate DHRs against RCM. Other influences may account as listed in a commentary by Chirumbolo (2013). For instance, a dose-dependent enhancement of CD63 expression upon prestimulation with interleukin $1 \beta$ was observed by Boehm et al., however, the authors also reported variation of this effect between different RCM. Summarizing, one can say literature has demonstrated sensitivities of $46-63 \%$ and specificities of $89-100 \%$ for BAT qualified on the combination of skin and provocation testing serving as the current diagnostic standard for RCM hypersensitivity. In general, good correlation between skin test, drug provocation test, and BAT has proven useful to complement in vivo testing (Pinnobphun et al., 2011; Salas et al., 2013). Nevertheless, only in rare cases drug-specific IgE is involved.

\section{Basophil Activation in Platinum-Containing Chemotherapeutic Hypersensitivity}

The DHRs against carboplatin or cisplatin, compounds used repeatedly at high doses in anti-cancer therapy, include hyperor hypotension, vomiting, dyspnea, and wheezing. They arise in up to $26.7 \%$ of patients and skin testing has been successfully performed although safety concerns exist regarding severe sideeffects for the patient and risk of exposure of the medical personnel (Markman et al., 1999; Leguy-Seguin et al., 2007;
Sugimoto et al., 2011). Consequently, the potential of BAT for predicting DHRs has been investigated using flow cytometric determination of CD203c (Iwamoto et al., 2012, 2014). These authors verified the involvement of IgE in carboplatin-induced DHR, as the basophil activation could be inhibited by wortmannin and anti-IgE pretreatment by omalizumab. In a case study of a severely anaphylactic history, BAT was applied evaluating the potential additive histamine-liberating effect by cisplatin following RCM administration (Viardot-Helmer et al., 2008). In conclusion, a similar situation to RCM seems to occur with platinum-containing anti-neoplastic drugs. In a low number of patients drug-specific IgE seems to be involved, still BAT may be suitable for diagnosis of severe immediate DHRs even if pharmacologic, i.e., non-immune-mediated mechanisms are underlying.

\section{Basophil Activation in Analgesic Hypersensitivity}

Non-steroidal anti-inflammatory drugs (NSAIDs) are known to frequently cause drug hypersensitivity reactions ranging from mild symptoms, e.g., skin reactions, to severe lifethreatening systemic complications (Kowalski et al., 2011). Although the symptoms can mimic typical IgE-mediated reactions, drug-specific IgE seems not to be involved in most NSAID hypersensitivities, as NSAID-specific IgE has only been detected for PP (Himly et al., 2003). Investigating severe selective diclofenac (DF) hypersensitivity using drug-human serum albumin conjugates of DF and the most common DF metabolites no drug-specific IgE was detectable by ELISA (Harrer et al., 2010). Instead of an IgE-mediated mechanism underlying immediate DHRs to DF, it has been hypothesized that the inhibition of cyclooxygenase-1, triggering the reduction of prostaglandin E2 accompanied by an increase in leukotriene production, accounts for the observed type I-like symptomatic (Mastalerz et al., 2004). Regardless the lack of NSAID-specific IgE, BAT has been explored by several groups leading to conflicting results (Gamboa et al., 2003a, 2004b; Celik et al., 2009; Gomez et al., 2009; Sanz et al., 2005; Malbran et al., 2007; Bavbek et al., 2009; Harrer et al., 2010; Korosec et al., 2011; Steiner et al., 2011; Abuaf et al., 2012; Kim and Cho, 2012; Hagau et al., 2013; Phillips-Angles et al., 2016). Sensitivities varied from 0 (Harrer et al., 2010; Steiner et al., 2011) to 80\% (Korosec et al., 2011) and specificities from 40 (Celik et al., 2009) to 100\% (Gamboa et al., 2003a, 2004b; Hagau et al., 2013). Reasons may be manifold as single NSAIDs and different combinations of NSAIDs were tested and activation times ranged from $15 \mathrm{~min}$ to $3 \mathrm{~h}$. In contrast to the negative BAT results, a most recent case report showed that basophils of an etoricoxib-hypersensitive patient were tested positive upon stimulation with the parent drug, although the authors excluded an involvement of drug specific IgE (PhillipsAngles et al., 2016). Here and in most other studies CD63 was the most commonly used activation marker. Introduction of CD203c by some groups (Bavbek et al., 2009; Celik et al., 2009; Abuaf et al., 2012) did not make results less ambiguous. Korosec et al. (2011) pointed out that a considerate pre-selection of most severe cases of DHRs may lead to an improvement in sensitivity and 
specificity of BAT. Another important factor for investigating NSAID hypersensitivity may be the time interval between the incident and BAT which should be less than 18 months according to the EAACI position paper on BAT (Hoffmann et al., 2015) or less than 6 months according to Gomez et al. (2009). To put it all in a nutshell, due to the many contradictory data regarding sensitivity and specificity, potentially resulting from differences in gating protocols influencing basophil activation (Chirumbolo, 2014), further large-scale trials following harmonized BAT protocols including data interpretation are needed to determine suitability of BAT for diagnosis of NSAID hypersensitivity.

\section{Basophil Activation in Hypersensitivity to Other Drugs and Some Remarks on Biologicals}

Various other drugs causing immediate DHRs have been tested using BAT including atropine (Cabrera-Freitag et al., 2009), glatiramer acetate (Soriano Gomis et al., 2012), methylprednisolone (Aranda et al., 2010; Ben Said et al., 2010a), omeprazole (Gamboa et al., 2003b), the diuretic hydrochlorothiazide (Gamboa et al., 2005; Manso et al., 2010), or antihistamines (Caceres Calle and Fernandez-Benitez, 2004; Bobadilla-Gonzalez et al., 2011; Lee et al., 2011; Sanchez Morillas et al., 2011) to name a few. However, these reports, most of them case studies, are hard to judge from the perspective of validating BAT performance, as no experimental details were presented. Aranda et al. (2010) showed log-shifts in CD63 expression in the dot plots and indicated involvement of methylprednisolone-specific IgE as activation could be inhibited by wortmannin. Accordingly, Soriano Gomis et al. (2012) determined drug-specific IgE in their workup, however, no details on how BAT was conducted were given. Unequivocal BAT performance with $100 \%$ sensitivity and $87.5 \%$ specificity evidenced by log-shifts in CD63 expression shown in dot plots was reported for Gelofusine ${ }^{\circledR}$ hypersensitivity of 6 patients (Apostolou et al., 2006).

Adverse reactions to biologicals are increasing due to their expanding utilization, as reviewed recently (Corominas et al., 2014; Galvao and Castells, 2015). For instance, rituximab hypersensitivity with an incidence of $5-10 \%$ has been evaluated using BAT in a cohort of $18 \mathrm{~B}$ cell lymphoma patients (Piva et al., 2012). The authors reported successful discrimination between patients and controls based on CD63 expression at in vivo concentrations, however, no experimental details were presented. Very recently an interesting new approach to predict DHRs against cetuximab during cancer immunotherapy based on evaluating the decrease in cetuximab molecules on basophils after dissociation of IgE from FceRI was reported (Iwamoto et al., 2016).
Notably, Werner J. Pichler has proposed a new classification for biologicals in five classes $(\alpha-\varepsilon)$, as biologicals differ in that they represent intact antigens themselves and are not metabolized like small molecular weight drugs (Pichler, 2006). We will thus not discuss ADRs against biologicals here.

\section{CONCLUSIONS}

Immediate DHRs present a complex phenomenon in regard to etiology. Similar clinical phenotypes are observed in "type I DHRs" with-according to the (pro-)hapten concept-drugspecific IgE against e.g., antibiotics, half of NMBAs, PP, or biologicals, and in "type I-like DHRs" elicited by alternative activation pathways as it is most likely the case with the other half of NMBAs, most RCM, platinum-containing chemotherapeutics, or NSAIDs. A close look on the CD63 upregulation pattern may help differentiating, as in type I DHRs the sudden basophil degranulation usually results in a log-shift of signal intensity. In contrast, no unequivocal conclusions on the underlying mechanism may be drawn when a "smear-like" CD63 upregulation is observed. In such cases, especially if basophil activation is weak, type I-like mechanisms independent of IgE cannot be excluded but bear the risk of false positive interpretation. We, therefore, recommend inclusion of plots/histograms in the publications to allow the reader evaluating the basophil activation profile. To circumvent bystander effects caused by interaction with other cells basophil purification protocols, as described recently (Steiner et al., 2016), may be considered, in particular when investigating more specific questions beyond diagnosis. Overall, the basophil response in BAT is influenced by many so far unpredictable factors which may impair the quality of results in certain cases (Chirumbolo, 2016).

Finally, we shall not forget that basophil activation test is an in vitro surrogate marker for a systemic reaction of the entire organism. This renders skin and/or provocation testing still of primary importance for diagnosis. Nevertheless, BAT has qualified a safe and suitable in vitro complement of in vivo testing in immediate DHRs, as most recently pointed out by a position paper of the European Network for Drug Allergy (ENDA) and the EAACI Drug Allergy Interest Group (Mayorga et al., 2016).

\section{AUTHOR CONTRIBUTIONS}

$\mathrm{MS}, \mathrm{AH}$, and $\mathrm{MH}$ were involved in the concept, literature screening, and writing of the article.

\section{ACKNOWLEDGMENTS}

This work was supported by grant P18820-B13 of the Austrian Science Funds (FWF). 


\section{REFERENCES}

Abuaf, N., Rostane, H., Barbara, J., Toly-Ndour, C., Gaouar, H., Mathelier-Fusade, P., et al. (2012). Comparison of CD63 upregulation induced by NSAIDs on basophils and monocytes in patients with NSAID hypersensitivity. J. Allergy (Cairo). 2012:580873. doi: 10.1155/2012/580873

Abuaf, N., Rostane, H., Rajoely, B., Gaouar, H., Autegarden, J. E., Leynadier, F., et al. (2008). Comparison of two basophil activation markers CD63 and $\mathrm{CD} 203 \mathrm{c}$ in the diagnosis of amoxicillin allergy. Clin. Exp. Allergy 38, 921-928. doi: 10.1111/j.1365-2222.2008.02960.x

Apostolou, E., Deckert, K., Puy, R., Sandrini, A., de Leon, M. P., Douglass, J. A., et al. (2006). Anaphylaxis to Gelofusine confirmed by in vitro basophil activation test: a case series. Anaesthesia 61, 264-268. doi: 10.1111/j.13652044.2005.04529.x

Aranda, A., Mayorga, C., Ariza, A., Dona, I., Blanca-Lopez, N., Canto, G., et al. (2010). IgE-mediated hypersensitivity reactions to methylprednisolone. Allergy 65, 1376-1380. doi: 10.1111/j.1398-9995.2010.02386.x

Aranda, A., Mayorga, C., Ariza, A., Dona, I., Rosado, A., Blanca-Lopez, N., et al. (2011). In vitro evaluation of IgE-mediated hypersensitivity reactions to quinolones. Allergy 66, 247-254. doi: 10.1111/j.1398-9995.2010.02460.x

Baldo, B. A., and Fisher, M. M. (1983). Detection of serum IgE antibodies that react with alcuronium and tubocurarine after life-threatening reactions to muscle-relaxant drugs. Anaesth. Intensive Care 11, 194-197.

Bavbek, S., Ikinciogullari, A., Dursun, A. B., Guloglu, D., Arikan, M., Elhan, A. H., et al. (2009). Upregulation of CD63 or CD203c alone or in combination is not sensitive in the diagnosis of nonsteroidal anti-inflammatory drug intolerance. Int. Arch. Allergy Immunol. 150, 261-270. doi: 10.1159/000222678

Ben Said, B., Berard, F., Bienvenu, J., Nicolas, J. F., and Rozieres, A. (2010b). Usefulness of basophil activation tests for the diagnosis of IgE-mediated allergy to quinolones. Allergy 65, 535-536. doi: 10.1111/j.1398-9995.2009.02213.x

Ben Said, B., Leray, V., Nicolas, J. F., Rozieres, A., and Berard, F. (2010a). Methylprednisolone-induced anaphylaxis: diagnosis by skin test and basophil activation test. Allergy 65, 531-532. doi: 10.1111/j.1398-9995.2009.02199.x

Blanca-Lopez, N., Ariza, A., Dona, I., Mayorga, C., Montañez, M. I., GarciaCampos, J., et al. (2013). Hypersensitivity reactions to fluoroquinolones: analysis of the factors involved. Clin. Exp. Allergy 43, 560-567. doi: $10.1111 /$ cea.12099

Bobadilla-Gonzalez, P., Perez-Rangel, I., Camara-Hijon, C., Garcia-Menaya, J. M., and Sanchez-Vega, S. (2011). Positive basophil activation test result in a patient with acute urticaria induced by cetirizine and desloratadine. Ann. Allergy Asthma Immunol. 106, 258-259. doi: 10.1016/j.anai.2010. 12.005

Bohm, I., Speck, U., and Schild, H. H. (2011). Pilot study on basophil activation induced by contrast medium. Fundam. Clin. Pharmacol. 25, 267-276. doi: 10.1111/j.1472-8206.2010.00826.x

Brockow, K., Romano, A., Aberer, W., Bircher, A. J., Barbaud, A., Bonadonna, P., et al. (2009). Skin testing in patients with hypersensitivity reactions to iodinated contrast media - a European multicenter study. Allergy 64, 234-241. doi: 10.1111/j.1398-9995.2008.01832.x

Cabrera-Freitag, P., Gastaminza, G., Goikoetxea, M. J., Lafuente, A., de la Borbolla, J. M., and Sanz, M. L. (2009). Immediate allergic reaction to atropine in ophthalmic solution confirmed by basophil activation test. Allergy 64, 1388-1389. doi: 10.1111/j.1398-9995.2009.02052.x

Caceres Calle, O., and Fernandez-Benitez, M. (2004). Allergy to dexchlorpheniramine. Study of a case. Allergol. Immunopathol. (Madr.) 32, 306-309. doi: 10.1016/S0301-0546(04)79259-9

Celik, G. E., Schroeder, J. T., Hamilton, R. G., Saini, S. S., and Adkinson, N. F. (2009). Effect of in vitro aspirin stimulation on basophils in patients with aspirin-exacerbated respiratory disease. Clin. Exp. Allergy 39, 1522-1531. doi: 10.1111/j.1365-2222.2009.03277.x

Chirumbolo, S. (2013). Basophil activation test (BAT) in the diagnosis of immediate hypersensitivity reactions to radiocontrast media. Allergy 68, 1627-1628. doi: 10.1111/all.12323

Chirumbolo, S. (2014). Major pitfalls in BAT performance may be caused by gating protocols and CD63\% cut off evaluation. Cytometry A 85, 382-385. doi: 10.1002/cyto.a.22466

Chirumbolo, S. (2016). Is basophil activation test (BAT) really useful for allergy diagnosis? Eur. Ann. Allergy Clin. Immunol. 48, 107-109.
Corominas, M., Gastaminza, G., and Lobera, T. (2014). Hypersensitivity reactions to biological drugs. J. Investig. Allergol. Clin. Immunol. 24, 212-225; quiz 211p following 225.

De Week, A. L., Sanz, M. L., Gamboa, P. M., Aberer, W., Sturm, G., Bilo, M. B., et al. (2009). Diagnosis of immediate-type beta-lactam allergy in vitro by flow-cytometric basophil activation test and sulfidoleukotriene production: a multicenter study. J. Investig. Allergol. Clin. Immunol. 19, 91-109.

Dvorak, A. M. (2005). Ultrastructural studies of human basophils and mast cells. J. Histochem. Cytochem. 53, 1043-1070. doi: 10.1369/jhc.5R6647.2005

Eberlein, B., Leon Suarez, I., Darsow, U., Rueff, F., Behrendt, H., and Ring, J. (2010). A new basophil activation test using CD63 and CCR3 in allergy to antibiotics. Clin. Exp. Allergy 40, 411-418. doi: 10.1111/j.13652222.2009.03426.x

Ebo, D. G., Bridts, C. H., Hagendorens, M. M., Mertens, C. H., De Clerck, L. S., and Stevens, W. J. (2006). Flow-assisted diagnostic management of anaphylaxis from rocuronium bromide. Allergy 61, 935-939. doi: 10.1111/j.1398-9995.2006.01094.x

Ebo, D. G., Hagendorens, M. M., Bridts, C. H., Schuerwegh, A. J., De Clerck, L. S., and Stevens, W. J. (2004). In vitro allergy diagnosis: should we follow the flow? Clin. Exp. Allergy 34, 332-339. doi: 10.1111/j.1365-2222.2004.01891.x

Ebo, D. G., Venemalm, L., Bridts, C. H., Degerbeck, F., Hagberg, H., De Clerck, L. S., et al. (2007). Immunoglobulin E antibodies to rocuronium: a new diagnostic tool. Anesthesiology 107, 253-259. doi: 10.1097/01.anes.0000270735.40872.f2

Galvao, V. R., and Castells, M. C. (2015). Hypersensitivity to biological agentsupdated diagnosis, management, and treatment. J. Allergy Clin. Immunol. Pract. 3, 175-185; quiz 186. doi: 10.1016/j.jaip.2014.12.006

Gamboa, P. M., Achotegui, V., Irigoyen, J., Perez-Asenjo, J., Merino, J., and Sanz, M. L. (2005). Hydrochlorothiazide-induced acute non-cardiogenic pulmonary edema. J. Investig. Allergol. Clin. Immunol. 15, 299-301.

Gamboa, P. M., Garcia-Aviles, M. C., Urrutia, I., Antepara, I., Esparza, R., and Sanz, M. L. (2004a). Basophil activation and sulfidoleukotriene production in patients with immediate allergy to betalactam antibiotics and negative skin tests. J. Investig. Allergol. Clin. Immunol. 14, 278-283.

Gamboa, P. M., Sanz, M. L., Caballero, M. R., Antepara, I., Urrutia, I., Jauregui, I., et al. (2003a). Use of CD63 expression as a marker of in vitro basophil activation and leukotriene determination in metamizol allergic patients. Allergy 58, 312-317. doi: 10.1034/j.1398-9995.2003.00096.x

Gamboa, P. M., Sanz, M. L., Urrutia, I., Jauregui, I., Antepara, I., Dieguez, I., et al. (2003b). CD63 expression by flow cytometry in the in vitro diagnosis of allergy to omeprazole. Allergy 58, 538-539. doi: 10.1034/j.1398-9995.2003.00155.x

Gamboa, P., Sanz, M. L., Caballero, M. R., Urrutia, I., Antepara, I., Esparza, R., et al. (2004b). The flow-cytometric determination of basophil activation induced by aspirin and other non-steroidal anti-inflammatory drugs (NSAIDs) is useful for in vitro diagnosis of the NSAID hypersensitivity syndrome. Clin. Exp. Allergy 34, 1448-1457. doi: 10.1111/j.1365-2222.2004.02050.x

Garcia-Ortega, P., and Marin, A. (2010). Usefulness of the basophil activation test (BAT) in the diagnosis of life-threatening drug anaphylaxis. Allergy 65, 1204. doi: 10.1111/j.1398-9995.2010.02333.x

Gomez, E., Blanca-Lopez, N., Torres, M. J., Requena, G., Rondon, C., Canto, G., et al. (2009). Immunoglobulin E-mediated immediate allergic reactions to dipyrone: value of basophil activation test in the identification of patients. Clin. Exp. Allergy 39, 1217-1224. doi: 10.1111/j.1365-2222.2009.03237.x

Hagau, N., Longrois, D., and Petrisor, C. (2013). Threshold for positivity and optimal dipyrone concentration in flow cytometry-assisted basophil activation test. Allergy Asthma Immunol. Res. 5, 383-388. doi: 10.4168/aair.2013.5.6.383

Harrer, A., Lang, R., Grims, R., Braitsch, M., Hawranek, T., Aberer, W., et al. (2010). Diclofenac hypersensitivity: antibody responses to the parent drug and relevant metabolites. PLoS ONE 5:e13707. doi: 10.1371/journal.pone.0013707

Hausmann, O. V., Gentinetta, T., Bridts, C. H., and Ebo, D. G. (2009). The basophil activation test in immediate-type drug allergy. Immunol. Allergy Clin. North Am. 29, 555-566. doi: 10.1016/j.iac.2009.04.011

Himly, M., Jahn-Schmid, B., Pittertschatscher, K., Bohle, B., Grubmayr, K., Ferreira, F., et al. (2003). IgE-mediated immediate-type hypersensitivity to the pyrazolone drug propyphenazone. J. Allergy Clin. Immunol. 111, 882-888. doi: $10.1067 /$ mai.2003.163

Hoffmann, H. J. (2015). News in cellular allergology: a review of the human mast cell and basophil granulocyte literature from January 2013 to May 2015. Int. Arch. Allergy Immunol. 168, 253-262. doi: 10.1159/000443960 
Hoffmann, H. J., Santos, A. F., Mayorga, C., Nopp, A., Eberlein, B., Ferrer, M., et al. (2015). The clinical utility of basophil activation testing in diagnosis and monitoring of allergic disease. Allergy 70, 1393-1405. doi: 10.1111/all.12698

Iwamoto, T., Hirai, H., Yamaguchi, N., Kobayashi, N., Sugimoto, H., Tabata, T., et al. (2014). Carboplatin-induced severe hypersensitivity reaction: role of IgEdependent basophil activation and FcepsilonRI. Cancer Sci. 105, 1472-1479. doi: $10.1111 /$ cas. 12538

Iwamoto, T., Okamoto, A., Ishinaga, H., Shimizu, K., Gayle, A. A., Arai, N., et al. (2016). A novel approach to predict cetuximab-induced hypersensitivity reaction: detection of drug-specific IgE on basophils. Cancer Med. doi: 10.1002/cam4.658. [Epub ahead of print].

Iwamoto, T., Yuta, A., Tabata, T., Sugimoto, H., Gabazza, E. C., Hirai, H., et al. (2012). Evaluation of basophil CD203c as a predictor of carboplatin-related hypersensitivity reaction in patients with gynecologic cancer. Biol. Pharm. Bull. 35, 1487-1495. doi: 10.1248/bpb.b12-00150

Javaloyes, G., Goikoetxea, M. J., Sanz, M. L., Cabrera-Freitag, P., and Gastaminza, G. (2012). Basophil activation test in the diagnosis of gadobutrol anaphylaxis. Ann. Allergy Asthma Immunol. 108, 286-287. doi: 10.1016/j.anai.2012.02.003

Katayama, H., Yamaguchi, K., Kozuka, T., Takashima, T., Seez, P., and Matsuura, K. (1990). Adverse reactions to ionic and nonionic contrast media. A report from the Japanese Committee on the Safety of Contrast Media. Radiology 175, 621-628. doi: 10.1148/radiology.175.3.2343107

Kim, M. S., and Cho, Y. J. (2012). Flow cytometry-assisted basophil activation test as a safe diagnostic tool for aspirin/NSAID hypersenstivity. Allergy Asthma Immunol. Res. 4, 137-142. doi: 10.4168/aair.2012.4.3.137

Korosec, P., Mavsar, N., Bajrovic, N., Silar, M., Mrhar, A., and Kosnik, M. (2011). Basophil responsiveness and clinical picture of acetylsalicylic acid intolerance. Int. Arch. Allergy Immunol. 155, 257-262. doi: 10.1159/000319846

Kowalski, M. L., Makowska, J. S., Blanca, M., Bavbek, S., Bochenek, G., Bousquet, J., et al. (2011). Hypersensitivity to nonsteroidal anti-inflammatory drugs (NSAIDs) - classification, diagnosis and management: review of the EAACI/ENDA and GA2LEN/HANNA. Allergy 66, 818-829. doi: 10.1111/j.1398-9995.2011.02557.x

Kvedariene, V., Kamey, S., Ryckwaert, Y., Rongier, M., Bousquet, J., Demoly, P., et al. (2006). Diagnosis of neuromuscular blocking agent hypersensitivity reactions using cytofluorimetric analysis of basophils. Allergy 61, 311-315. doi: 10.1111/j.1398-9995.2006.00978.x

Lee, S. W., Byun, J. Y., Choi, Y. W., Myung, K. B., and Choi, H. Y. (2011). Fexofenadine-induced urticaria. Ann. Dermatol. 23, S329-S332. doi: 10.5021/ad.2011.23.3.329

Leguy-Seguin, V., Jolimoy, G., Coudert, B., Pernot, C., Dalac, S., Vabres, P., et al. (2007). Diagnostic and predictive value of skin testing in platinum salt hypersensitivity. J. Allergy Clin. Immunol. 119, 726-730. doi: 10.1016/j.jaci.2006.11.640

Leysen, J., Bridts, C. H., De Clerck, L. S., Vercauteren, M., Lambert, J., Weyler, J. J., et al. (2011). Allergy to rocuronium: from clinical suspicion to correct diagnosis. Allergy 66, 1014-1019. doi: 10.1111/j.1398-9995.2011.02569.x

Leysen, J., De Witte, L., Sabato, V., Faber, M., Hagendorens, M., Bridts, C., et al. (2013). IgE-mediated allergy to pholcodine and cross-reactivity to neuromuscular blocking agents: lessons from flow cytometry. Cytometry B Clin. Cytom. 84, 65-70. doi: 10.1002/cyto.b.21074

Lieberman, P. L., and Seigle, R. L. (1999). Reactions to radiocontrast material. Anaphylactoid events in radiology. Clin. Rev. Allergy Immunol. 17, 469-496. doi: $10.1007 / \mathrm{BF} 02737651$

Lobera, T., Audicana, M. T., Alarcon, E., Longo, N., Navarro, B., and Munoz, D. (2010). Allergy to quinolones: low cross-reactivity to levofloxacin. J. Investig. Allergol. Clin. Immunol. 20, 607-611.

MacGlashan, D. Jr. (2010). Expression of CD203c and CD63 in human basophils: relationship to differential regulation of piecemeal and anaphylactic degranulation processes. Clin. Exp. Allergy 40, 1365-1377. doi: 10.1111/j.13652222.2010.03572.x

MacGlashan, D. W. Jr. (2013). Basophil activation testing. J. Allergy Clin. Immunol. 132, 777-787. doi: 10.1016/j.jaci.2013.06.038

Malbran, A., Yeyati, E., Rey, G. L., and Galassi, N. (2007). Diclofenac induces basophil degranulation without increasing CD63 expression in sensitive patients. Clin. Exp. Immunol. 147, 99-105. doi: 10.1111/j.13652249.2006.03265.x
Manfredi, M., Severino, M., Testi, S., Macchia, D., Ermini, G., Pichler, W. J., et al. (2004). Detection of specific IgE to quinolones. J. Allergy Clin. Immunol. 113, 155-160. doi: 10.1016/j.jaci.2003.09.035

Mangodt, E. A., Van Gasse, A. L., Decuyper, I., Uyttebroek, A., Faber, M. A., Sabato, V., et al. (2015). In vitro diagnosis of immediate drug hypersensitivity: should we go with the flow. Int. Arch. Allergy Immunol. 168, 3-12. doi: $10.1159 / 000440663$

Manso, L., Heili, S., Fernandez-Nieto, M., Sastre, B., and Sastre, J. (2010). Basophil activation in two cases of hydrochlorothiazide-induced noncardiogenic pulmonary edema. Allergy 65, 135-136. doi: 10.1111/j.1398-9995.2009.02149.x

Markman, M., Kennedy, A., Webster, K., Elson, P., Peterson, G., Kulp, B., et al. (1999). Clinical features of hypersensitivity reactions to carboplatin. J. Clin. Oncol. 17, 1141.

Mastalerz, L., Setkowicz, M., Sanak, M., and Szczeklik, A. (2004). Hypersensitivity to aspirin: common eicosanoid alterations in urticaria and asthma. J. Allergy Clin. Immunol. 113, 771-775. doi: 10.1016/j.jaci.2003.12.323

Mayorga, C., Andreu, I., Aranda, A., Dona, I., Montañez, M. I., Blanca-Lopez, N., et al. (2013). Fluoroquinolone photodegradation influences specific basophil activation. Int. Arch. Allergy Immunol. 160, 377-382. doi: 10.1159/000343023

Mayorga, C., Celik, G., Rouzaire, P., Whitaker, P., Bonadonna, P., Cernadas, J. R., et al. (2016). In vitro tests for drug hypersensitivity reactions. An ENDA/EAACI drug allergy interest group position paper. Allergy. doi: 10.1111/all.12886. [Epub ahead of print].

McGowan, E. C., and Saini, S. (2013). Update on the performance and application of basophil activation tests. Curr. Allergy Asthma Rep. 13, 101-109. doi: 10.1007/s11882-012-0324-x

Monneret, G., Benoit, Y., Debard, A. L., Gutowski, M. C., Topenot, I., and Bienvenu, J. (2002). Monitoring of basophil activation using CD63 and CCR3 in allergy to muscle relaxant drugs. Clin. Immunol. 102, 192-199. doi: 10.1006/clim.2001.5156

Monneret, G., Benoit, Y., Gutowski, M. C., and Bienvenu, J. (2000). Detection of basophil activation by flow cytometry in patients with allergy to musclerelaxant drugs. Anesthesiology 92, 275-277. doi: 10.1097/00000542-20000100000044

Naisbitt, D. J., Gordon, S. F., Pirmohamed, M., and Park, B. K. (2000). Immunological principles of adverse drug reactions: the initiation and propagation of immune responses elicited by drug treatment. Drug Saf. 23, 483-507. doi: 10.2165/00002018-200023060-00002

Park, B. K., Pirmohamed, M., and Kitteringham, N. R. (1998). Role of drug disposition in drug hypersensitivity: a chemical, molecular, and clinical perspective. Chem. Res. Toxicol. 11, 969-988. doi: 10.1021/tx980058f

Philipse, E., Sabato, V., Bridts, C., De Clerck, L., and Ebo, D. (2013). Basophil activation in the diagnosis of life-threatening hypersensitivity reaction to iodinated contrast media: a case report. Acta Clin. Belg. 68, 140-142. doi: 10.2143/ACB. 3251

Phillips-Angles, E., Gonzalez-Munoz, M., Dominguez-Ortega, J., Cabanas, R., and Quirce, S. (2016). Usefulness of basophil activation test in diagnosis of hypersensitivity to etoricoxib. J. Allergy Clin. Immunol. Pract. 4, 350-351. doi: 10.1016/j.jaip.2015.11.008

Pichler, W. J. (2003). Delayed drug hypersensitivity reactions. Ann. Intern. Med. 139, 683-693. doi: 10.7326/0003-4819-139-8-200310210-00012

Pichler, W. J. (2006). Adverse side-effects to biological agents. Allergy 61, 912-920. doi: 10.1111/j.1398-9995.2006.01058.x

Pichler, W. J., Adam, J., Watkins, S., Wuillemin, N., Yun, J., and Yerly, D. (2015). Drug hypersensitivity: how drugs stimulate t cells via pharmacological interaction with immune receptors. Int. Arch. Allergy Immunol. 168, 13-24. doi: $10.1159 / 000441280$

Pichler, W. J., Beeler, A., Keller, M., Lerch, M., Posadas, S., Schmid, D., et al. (2006). Pharmacological interaction of drugs with immune receptors: the p-i concept. Allergol. Int. 55, 17-25. doi: 10.2332/allergolint.55.17

Pichler, W. J., Naisbitt, D. J., and Park, B. K. (2011). Immune pathomechanism of drug hypersensitivity reactions. J. Allergy Clin. Immunol. 127, S74-81. doi: 10.1016/j.jaci.2010.11.048

Pinnobphun, P., Buranapraditkun, S., Kampitak, T., Hirankarn, N., and Klaewsongkram, J. (2011). The diagnostic value of basophil activation test in patients with an immediate hypersensitivity reaction to radiocontrast media. Ann. Allergy Asthma Immunol. 106, 387-393. doi: 10.1016/j.anai.2010.12.020 
Piva, E., Chieco-Bianchi, F., Krajcar, V., Aversa, S., and Plebani, M. (2012). Adverse reactions in patients with B-cell lymphomas during combined treatment with rituximab: in vitro evaluation of rituximab hypersensitivity by basophil activation test. Am. J. Hematol. 87, E130-E131. doi: 10.1002/ajh.23329

Rouzaire, P., Nosbaum, A., Denis, L., Bienvenu, F., Berard, F., Cozon, G., et al. (2012). Negativity of the basophil activation test in quinolone hypersensitivity: a breakthrough for provocation test decision-making. Int. Arch. Allergy Immunol. 157, 299-302. doi: 10.1159/000328211

Sainte-Laudy, J., Boumediene, A., Touraine, F., Orsel, I., and Cogne, M. (2006). Analysis of IgE down regulation induced by basophil activation. Application to the diagnosis of muscle relaxant allergic hypersensitivity by flow cytometry. Inflamm. Res. 55(Suppl. 1), S21-S22. doi: 10.1007/s00011-005-0023-3

Salas, M., Gomez, F., Fernandez, T. D., Dona, I., Aranda, A., Ariza, A., et al. (2013). Diagnosis of immediate hypersensitivity reactions to radiocontrast media. Allergy 68, 1203-1206. doi: 10.1111/all.12214

Sanchez Morillas, L., Rojas Perez-Ezquerra, P., Reano Martos, M., Sanz, M. L., and Laguna Martinez, J. J. (2011). Urticaria due to antihistamines. J. Investig. Allergol. Clin. Immunol. 21, 66-68.

Sanz, M. L., Gamboa, P., and de Weck, A. L. (2005). A new combined test with flowcytometric basophil activation and determination of sulfidoleukotrienes is useful for in vitro diagnosis of hypersensitivity to aspirin and other nonsteroidal anti-inflammatory drugs. Int. Arch. Allergy Immunol. 136, 58-72. doi: $10.1159 / 000082586$

Sanz, M. L., Gamboa, P. M., Antepara, I., Uasuf, C., Vila, L., Garcia-Aviles, C., et al. (2002). Flow cytometric basophil activation test by detection of CD63 expression in patients with immediate-type reactions to betalactam antibiotics. Clin. Exp. Allergy 32, 277-286. doi: 10.1046/j.1365-2222.2002.01305.x

Seitz, C. S., Brocker, E. B., and Trautmann, A. (2009). Diagnostic testing in suspected fluoroquinolone hypersensitivity. Clin. Exp. Allergy 39, 1738-1745. doi: 10.1111/j.1365-2222.2009.03338.x

Soriano Gomis, V., Perez Sempere, A., Gonzalez Delgado, P., Sempere, J. M., Niveiro Hernandez, E., and Marco, F. M. (2012). Glatiramer acetate anaphylaxis: detection of antibodies and basophil activation test. J. Investig. Allergol. Clin. Immunol. 22, 65-66.

Steiner, M., Harrer, A., Lang, R., Schneider, M., Ferreira, T., Hawranek, T., et al. (2011). Basophil activation test for investigation of IgE-mediated mechanisms in drug hypersensitivity. J. Vis. Exp. e3263. doi: 10.3791/3263

Steiner, M., Hawranek, T., Schneider, M., Ferreira, F., Horejs-Hoeck, J., Harrer, A., et al. (2016). Elevated toll-like receptor-induced CXCL8 secretion in human blood basophils from allergic donors is independent of toll-like receptor expression levels. PLoS ONE 11:e0149275. doi: 10.1371/journal.pone.0149275

Steiner, M., Wedi, B., Harrer, A., Schneider, M., Ferreira, F., and Himly, M. (2014). Three Further Cases Corroborate Lack of IgE in Selective Severe ProvocationConfirmed Diclofenac Hypersensitivity. Pisa: Pacini Editore, S.p.A.

Stellato, C., de Crescenzo, G., Patella, V., Mastronardi, P., Mazzarella, B., and Marone, G. (1996). Human basophil/mast cell releasability. XI. Heterogeneity of the effects of contrast media on mediator release. J. Allergy Clin. Immunol. 97, 838-850. doi: 10.1016/S0091-6749(96)80162-X

Sudheer, P. S., and Appadurai, I. R. (2007). Anaphylaxis to vecuronium: the use of basophil CD63 expression as a possible screening tool to identify a safe alternative. J. Clin. Anesth. 19, 555-557. doi: 10.1016/j.jclinane.2007.03.011

Sudheer, P. S., Hall, J. E., Read, G. F., Rowbottom, A. W., and Williams, P. E. (2005). Flow cytometric investigation of peri-anaesthetic anaphylaxis using
CD63 and CD203c. Anaesthesia 60, 251-256. doi: 10.1111/j.1365-2044.2004. 04086.x

Sugimoto, H., Iwamoto, T., Murashima, Y., Tabata, T., Sagawa, N., and Okuda, M. (2011). Risk factors contributing to the development of carboplatinrelated delayed hypersensitivity reactions in Japanese patients with gynecologic cancers. Cancer Chemother. Pharmacol. 67, 415-419. doi: 10.1007/s00280-0101338-5

Torres, M. J., Ariza, A., Fernandez, J., Moreno, E., Laguna, J. J., Montañez, M. I., et al. (2010a). Role of minor determinants of amoxicillin in the diagnosis of immediate allergic reactions to amoxicillin. Allergy 65, 590-596. doi: 10.1111/j.1398-9995.2009.02245.x

Torres, M. J., Ariza, A., Mayorga, C., Dona, I., Blanca-Lopez, N., Rondon, C., et al. (2010b). Clavulanic acid can be the component in amoxicillin-clavulanic acid responsible for immediate hypersensitivity reactions. J. Allergy Clin. Immunol. 125, 502.e2-505.e2. doi: 10.1016/j.jaci.2009.11.032

Torres, M. J., Montañez, M. I., Ariza, A., Salas, M., Fernandez, T. D., Barbero, N., et al. (2016). The role of $\operatorname{IgE}$ recognition in allergic reactions to amoxicillin and clavulanic acid. Clin. Exp. Allergy 46, 264-274. doi: 10.1111/cea. 12689

Torres, M. J., Padial, A., Mayorga, C., Fernandez, T., Sanchez-Sabate, E., CornejoGarcia, J. A., et al. (2004). The diagnostic interpretation of basophil activation test in immediate allergic reactions to betalactams. Clin. Exp. Allergy 34, 1768-1775. doi: 10.1111/j.1365-2222.2004.02110.x

Torres, M. J., Romano, A., Blanca-Lopez, N., Dona, I., Canto, G., Ariza, A., et al. (2011). Immunoglobulin E-mediated hypersensitivity to amoxicillin: in vivo and in vitro comparative studies between an injectable therapeutic compound and a new commercial compound. Clin. Exp. Allergy 41, 1595-1601. doi: 10.1111/j.1365-2222.2011.03834.x

Trcka, J., Schmidt, C., Seitz, C. S., Brocker, E. B., Gross, G. E., and Trautmann, A. (2008). Anaphylaxis to iodinated contrast material: nonallergic hypersensitivity or IgE-mediated allergy? AJR Am. J. Roentgenol. 190, 666-670. doi: 10.2214/AJR.07.2872

Vervloet, D., Nizankowska, E., Arnaud, A., Senft, M., Alazia, M., and Charpin, J. (1983). Adverse reactions to suxamethonium and other muscle relaxants under general anesthesia. J. Allergy Clin. Immunol. 71, 552-559. doi: 10.1016/00916749(83)90436-0

Viardot-Helmer, A., Ott, H., Sauer, I., and Merk, H. F. (2008). [Basophil activation test as in vitro assay for cisplatin allergy]. Hautarzt 59, 883-884. doi: 10.1007/s00105-008-1653-5

Wolf, G. L., Arenson, R. L., and Cross, A. P. (1989). A prospective trial of ionic vs. nonionic contrast agents in routine clinical practice: comparison of adverse effects. AJR Am. J. Roentgenol. 152, 939-944. doi: 10.2214/ajr.152.5.939

Conflict of Interest Statement: The authors declare that the research was conducted in the absence of any commercial or financial relationships that could be construed as a potential conflict of interest.

Copyright (c) 2016 Steiner, Harrer and Himly. This is an open-access article distributed under the terms of the Creative Commons Attribution License (CC BY). The use, distribution or reproduction in other forums is permitted, provided the original author(s) or licensor are credited and that the original publication in this journal is cited, in accordance with accepted academic practice. No use, distribution or reproduction is permitted which does not comply with these terms. 